\title{
Processing of Instantaneous Angular Speed Signal for Detection of a Diesel Engine Failure
}

\author{
Adam Charchalis and Mirosław Dereszewski \\ Faculty of Marine Engineering, Gdynia Maritime University, 81-87 Morska Street, \\ 81-225 Gdynia, Poland \\ Correspondence should be addressed to Mirosław Dereszewski; deresz1@o2.pl
}

Received 20 February 2013; Accepted 3 April 2013

Academic Editor: Henryk Śniegocki

Copyright ( 2013 A. Charchalis and M. Dereszewski. This is an open access article distributed under the Creative Commons Attribution License, which permits unrestricted use, distribution, and reproduction in any medium, provided the original work is properly cited.

\begin{abstract}
Continuous monitoring of diesel engine performance under its operating is critical for the prediction of malfunction development and subsequently functional failure detection. Analysis of instantaneous angular speed (IAS) of the crankshaft is considered as one of the nonintrusive and effective methods of the detection of combustion quality deterioration. In this paper results of experimental verification of fuel system's malfunction detecting, using optical encoder for IAS recording are presented. The implemented method relies on the comparison of measurement results, recorded under healthy and faulty conditions of the engine. Elaborated dynamic model of angular speed variations enables us to build templates of engine behavior. Recorded during experiment, values of cylinder pressure were taken for the approximation of pressure basic waveform. The main task of data processing is smoothing the raw angular speed signal. The noise is due to sensor mount vibrations, signal emitter machining, engine body vibrations, and crankshaft torsional vibrations. Smoothing of the measurement data was carried out by the implementation of the Savitzky-Golay filter. Measured signal after smoothing was compared with the model of IAS run.
\end{abstract}

\section{Introduction}

Diesel engines are one of the most critical mechanisms having impact on safety of shipping. Unpredicted failures of engines, installed on board as main propulsion or electrogenerator units, can result in serious consequences, jeopardizing human life and environment. One of the most common problems occurring during diesel engines operation are malfunctions of fuel injection systems. Faults developed in high pressure fuel systems will directly affect combustion process and deteriorate engine performance. Engine misfire, increasing of $\mathrm{NO}_{x}$ and $\mathrm{SO}_{x}$ contents in exhaust gases, and higher vibrations level are typical consequences of fuel system malfunction. To prevent the occurrence of unpredicted engine failures or its exploiting at low efficiency and high pollutants emission level, continuous monitoring of engine performance and deviations from normal state is necessary.

The most common method of engine state diagnosis is measurement of in-cylinder pressure. This technique can provide a direct indication of engine performance and the state of combustion process. Unfortunately, due to intrusive nature of the method, its application is limited to the engines equipped with indicator cocks (mostly medium and low speed diesel engines). Moreover, because of high temperature and soot containment of exhaust smoke, expensive and delicate pressure sensors cannot be exposed at exhaust gases action for long time. The above facts are the reason of limitations of MIP (Mean Indicated Pressure) employment as a continuous engine monitoring. Considered as nonintrusive method, measurement of instantaneous angular speed, due to significant development of data acquisition and processing, has gained broad spectrum of applications for condition monitoring. IAS technique was used for cylinder pressure reconstruction [1], monitoring of gear transmission [2], fault detection of high speed diesel engines [3], and multicylinder diesel engines driving electrogenerator sets $[4,5]$. Instantaneous angular speed analysis can be used for monitoring of engine performance because oscillations of crankshaft angular speed reflects directly gas pressure torque produced by piston-conrod assembly during combustion process. 


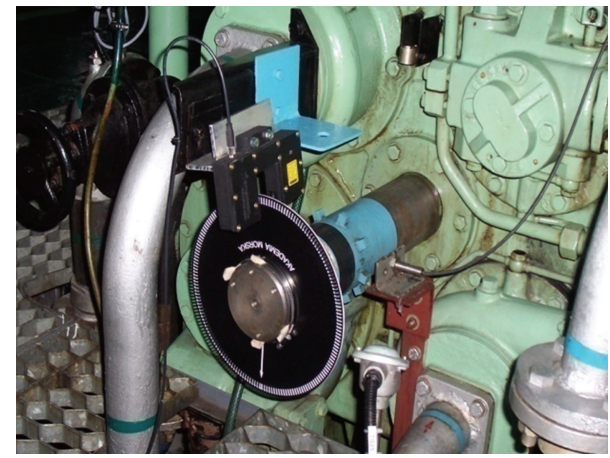

(a)

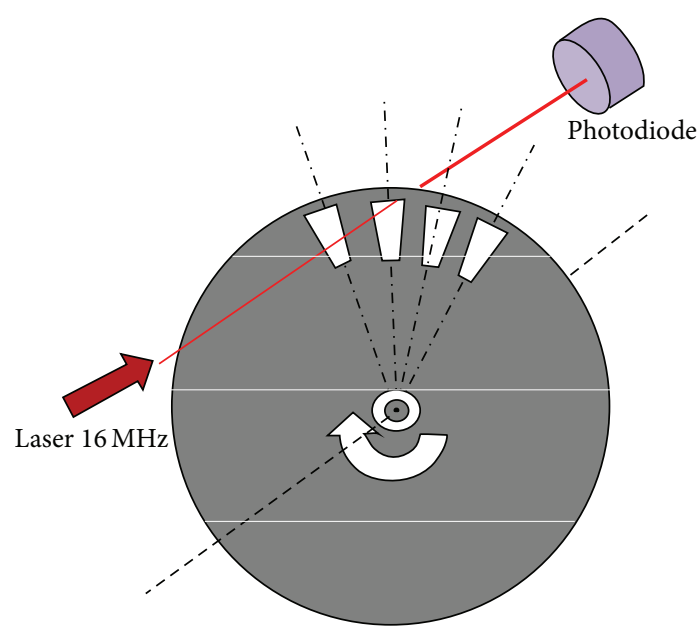

(b)

FIGURE 1: Encoder installed at free end of the shaft and scheme of speed measurement.

TABLE 1: Test engine particulars.

\begin{tabular}{lc}
\hline Manufacturer & HCP Cegielski/Sulzer \\
Type & 3 AL 25/30 \\
Rated power $(\mathrm{kW})$ & 408 \\
Cylinder number & 3 \\
Cylinder swept capacity $\left(\mathrm{cm}^{3}\right)$ & 4922 \\
Rotational speed (rpm) & 750 \\
Compression ratio & $13: 1$ \\
\hline
\end{tabular}

For IAS analysis and fault diagnosis, itis useful to create a model of dynamic system aimed at the simulation of angular speed variations of healthy and nonhealthy diesel engine. Two degrees of freedom, simple dynamic model, enables us to predict angular speed fluctuation of three-cylinder fourstroke diesel engine. The angular speed is strongly affected by tangential force coming from gas pressure and vertical imbalance inertial forces induced by reciprocating masses of piston and connecting rod. The character of acting forces lets us assume that IAS can be utilized for detecting engine faults related to combustion process changes, that is, high pressure fuel system leakage. IAS technique allows not only fault detection but also identification of faulty cylinder. Charles et al. [6] propose presenting the IAS waveform in polar coordinate form, Gawande et al. [7] developed the technique based on analysis of the lowest three harmonics orders, and Desbazeille et al. [5] presented the method of comparison of angular speed difference between healthy and faulty condition. The last one was used in our experiment.

For instantaneous angular speed measurements, the method based on optical encoder was implemented. That method is considered as more practical due to its simplicity and being less likely to be affected by ambient interference. Recently several approaches to the noise reduction subject have been presented [8]. This paper presents the method of raw signal optimizing using moving polynomial approximation (Savitzky-Golay filter). The instantaneous angular speed signal after noise decomposition is compared with angular speed runs based on the simplified model. Comparison of both is a base for failure detection and identification.

\section{Test Rig Description}

Simulation of diesel engine fault was carried out at test rig of Maritime University in Gdynia. Test engine was threecylinder, four-stroke medium speed marine diesel driving three-phase electrogenerator. Main particulars of the engine are presented in Table 1.

For measurement of instantaneous angular speed, the optical encoder was installed at free end of the crankshaft. The encoder has 180 circumferential spaced slits, with resolution of $2^{\circ}$. The errors of manufacturing can be omitted. The counting system ETNP-10 records numbers of impulses of laser beam going through the slit and blind when tooth is passing through. The laser head emits signals with frequency of $16 \mathrm{MHz}$, which ensures minimizing of IAS measurement errors according to (1) which was presented in [9]:

$$
f_{s}>4\left[n f_{\text {shaft }}+\left(n_{h} f_{\text {shaft }}+n \Delta f\right)\right],
$$

where $f_{s}$ is the sampling frequency, $n$ is the number of slits of encoder, $n_{h}$ is the highest order of IAS concerned, $f_{\text {shaft }}$ is the shaft rotating frequency, and $\Delta f$ is the estimated shaft speed variation.

In this case, where number of slits was 180, shaft frequency was $12.5 \mathrm{~Hz}$, highest order concerned was 23, and estimated shaft speed variation was $0.15 \mathrm{~Hz}$; required minimum sampling frequency calculated using (1) was $85 \mathrm{kHz}$.

The encoder disk has one nonproportional slit which plays a role of trigger when 1st cylinder is in TDC. The encoder disc installed at free end of the shaft and the functional scheme of the encoder are presented in Figure 1.

Simulation of faulty condition in form of injection pump leakage was conducted in way of release the discharge bolt in the pump, what results in opening of draining pass-by duct inside the pump. 


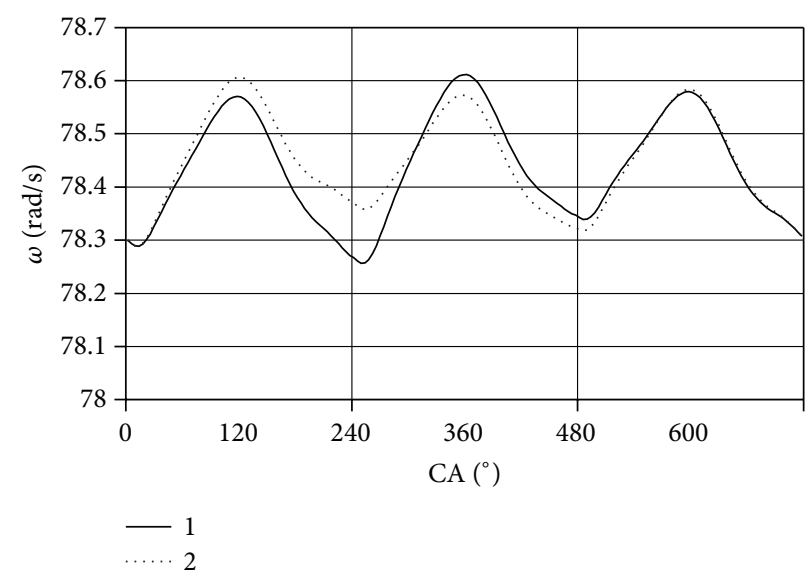

Figure 2: IAS waveforms of healthy engine (solid line 1) and fuel leakage (dotted line 2).

\section{Engine Model}

For modeling purposes one has to assume that the crankshaft is sufficiently rigid, the load torque is constant, and the engine is decoupled from the load; thus the engine dynamics can be described by (2). For model purposes, $T_{\text {fr }}$ was taken as a lumped part of load torque:

$$
J_{E}(\varphi) \frac{d^{2} \varphi}{d t^{2}}=T_{\text {gas }}-T_{\text {mas }}-\left(T_{\text {load }}+T_{\text {fr }}\right),
$$

where $J_{E}$ is the mass inertia on engine side and $T_{\text {gas }}, T_{\text {mas }}$, $T_{\text {load }}$, and $T_{\text {fr }}$ are, respectively, the gas, mass, load, and friction torques. The gas torque, derived from in-cylinder pressure, was calculated according to (3). Values of gas pressure in domain of crankshaft angle were directly measured in health condition and with fault simulation:

$$
T_{\mathrm{gas}}=P * A * 0.5 s\left[\sin \theta+\frac{\sin 2 \theta}{2 \sqrt{n^{2}-\sin ^{2} \theta}}\right],
$$

where $P$ is the in-cylinder pressure, $A$ is the piston area, $s$ is the piston stroke, and $\theta$ is the crank angle. Torque created by inertia forces of masses of pistons, connecting rods and cranks in reciprocating movement, for three-cylinder engines can be calculated by

$$
T_{\text {mas }}=m_{p} r \omega^{2}\left(-\frac{9}{4} \lambda \sin 3 \theta+\frac{9}{32} \lambda^{4} \sin 6 \theta\right) .
$$

Friction torque was calculated experimentally, in way of measurement of mean effective pressure when engine was working without load.

3.1. Model Simulation of Engine Failure. The waveforms of IAS of healthy and faulty conditions were built basing on engine dynamic model and recorded real pressure values. In Figure 2 waveforms of model-based IAS values of engine healthy and faulty running are presented.

In order to evaluate the value of standard deviation of IAS per engine cycle as a parameter of failure condition

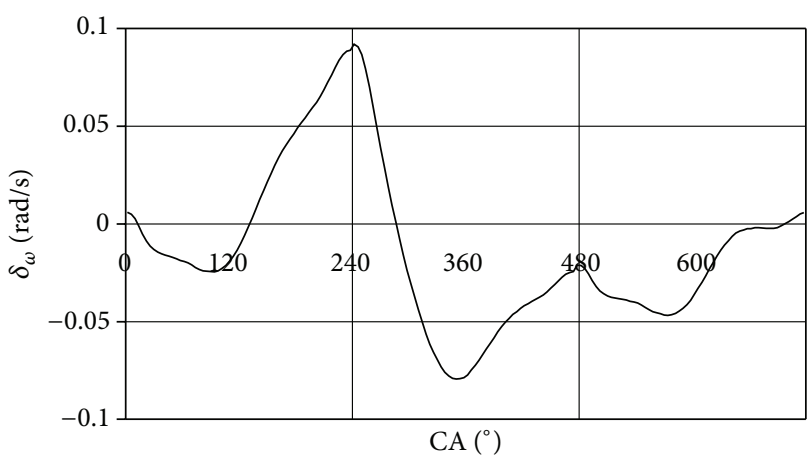

FIGURE 3: Sample of waveform of difference of relative angular speed between healthy and faulty engines.

detection, standard deviation for healthy, $\sigma_{\text {health }}$, and faulty, $\sigma_{\text {fault }}$, engines was calculated by

$$
\sigma=\sqrt{\frac{1}{n} \sum_{j=1}^{n}\left(\omega_{j}-\bar{\omega}\right)^{2}}
$$

The results $-\sigma_{\text {health }}=0,10541, \sigma_{\text {fault }}=0,10829$-indicate that the standard deviation does not bear any useful information about changes of engine condition.

Afterwards, curves of difference between IAS relative values of healthy and fault states were created, in domain of crank angle. The run of differential value was assumed as an indicator of failure detection and localization.

Analysis of changes of difference value between health engine's waveform and fuel leakage state gives more information which may have application in practical cases. As presented in Figure 3, the difference factor decreases significantly in angular zone of $240^{\circ}$ to $360^{\circ}$, during working stroke of 2nd (affected) cylinder.

Model-based simulations' waveforms are the basic form for comparison with waveforms built on results of IAS measurement, which is presented in the next section.

\section{Measurement Results}

The raw signal was in form of group of numbers of impulses related to time when slit and toothwerepassing through the laser beam. The length of data record was 10 subsequent revolutions, what was 5 firing cycles of 4 -stroke engine. For every load and simulation, three subsequent records were taken. The couple "slit" and "tooth" angular width is $2^{\circ}=$ $0,0349 \mathrm{rad}$; thus instantaneous angular speed $\omega_{i}$ is calculate according to

$$
\omega_{i}=\frac{\Delta \varphi}{\Delta \tau}=\frac{\Delta \varphi * f_{s}}{z_{i}}=\frac{0,0349 * 16 * 10^{6}}{z_{i}},
$$

where $z_{i}$ is the number of impulses recorded for slit-tooth couple $i$. 


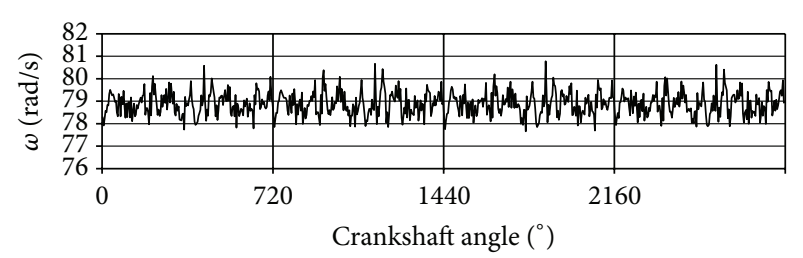

(a)

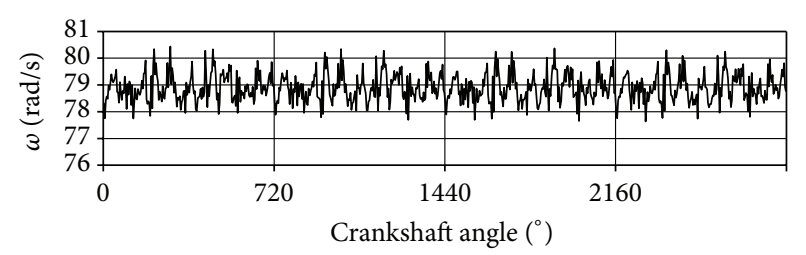

(b)

FIGURE 4: Noisy data IAS signal: (a) healthy engine, (b) failure of injection pump.

TABLE 2: Standard deviation per cycle of IAS.

\begin{tabular}{lccc}
\hline & 1st cycle & 2nd cycle & 3rd cycle \\
\hline Healthy engine & 0,499 & 0,542 & 0,524 \\
Fault condition & 0,524 & 0,523 & 0,532 \\
\hline
\end{tabular}

In Figure 4 run of noisy data of angular speed recorded for healthy and faulty conditions is presented.

The standard deviation per engine cycle of instantaneous angular speed fluctuation was calculated according to (2) and is shown in Table 2. One has to see that deviation value $\sigma$ does not bear any information about engine condition deterioration.

Desbazeille et al. [5] shows the comparison of relative angular speed value waveform as a useful method of fault detection. That method relies on the calculation of difference between instantaneous speed in faulty and healthy conditions expressed by

$$
\Delta \omega_{r i}=\frac{\omega_{1 i}}{\bar{\omega}_{1}}-\frac{\omega_{0 i}}{\bar{\omega}_{0}}
$$

where $\omega_{1}$ is the angular speed of fault condition and $\omega_{0}$ is the angular speed of healthy engine.

To apply the above formula, the synthetic noisy signal has to be treated by noise decomposition process. Methods of least-squares smoothing of angular speed signal were studied by Dereszewski et al. [10], who showed that moving approximation by digital smoothing polynomial (SavitzkyGolay filter) is a good tool for IAS noise decomposition.

The idea of Savitzky-Golay filter smoothing is to obtain, for sequence of samples $x[n]$ of signals, for a group of $2 M+$ 1 samples with the centre at $n=0$, the coefficients of a polynomial represented by

$$
p_{n}=\sum_{j}^{N} a_{j} n^{j}
$$

in a way to minimize the mean-squared approximation error for group of samples calculated according to

$$
\varepsilon=\sum_{n=-M}^{M}(p(n)-x[n])^{2}
$$

within determination in every center of interval point $n$ of measurement series, an approximation value of moving approximation interval with $2 M+1$ width. The normal equations for least-squares approximation problem is a set of $N+1$ equations in $N+1$ unknowns meeting equation

$$
\sum_{j=0}^{N}\left(\sum_{n=-M}^{M} n^{i+j}\right) a_{k}=\sum_{n=-M}^{M} n^{i} x[n], \quad i=0,1, \ldots, N .
$$

For smoothing of recorded waveforms, the moving approximation by polynomial degree $N=3$ was implemented. The width of intervals and the polynomial degree have an impact on results of smoothing. Higher degree preserve better narrow profiles of smoothed data, but gives some loses of broad features. In order to get best fitting, the raw signal was treated by several filters with different point numbers. Comparison of results pointed the most fitting curve, which was a result of triple repeated decomposition, with three subsequent filters, with intervals $2 M+1=$ $\{8,24,36\}$. Obtained in that way smooth line profiles were superposed with model ones, in order to evaluate the level of conformity. Expected outcome from comparison was to answer two essential questions: whether simulated values of speed difference can be a template for classification of measurement's results of real condition, and if simulation of good health waveform can be used as a substitution of healthy engine angular speed waveform. The healthy engine signal waveform after noise decomposition in comparison to model waveform of healthy engine's run is presented in Figure 5. Similar comparison for faulty engine is presented in Figure 6.

Pearson's Coefficient of Correlation was calculated for subsequent zones of crank angle, with width $120^{\circ}$, in order to evaluate similarity of waveforms shapes. High level of conformity is very important for future analysis, when model of healthy engine speed will be compared with real IAS waveform, based on data measured at engine with failure.

High level of conformity lets us assume that model simulation of changes of engine condition should reflect real angular speed behavior.

\section{Failure Detection}

The first step to the detection of failure was the calculation using (11) of a difference between values of relative angular speed of good and fault conditions of engine:

$$
\delta_{\phi i}=\omega_{\mathrm{rf}}-\omega_{\mathrm{rh}}
$$

where $\omega_{\text {rf }}$ is the relative angular speed of faulty engine and $\omega_{\text {rh }}$ is the relative angular speed of healthy engine. 


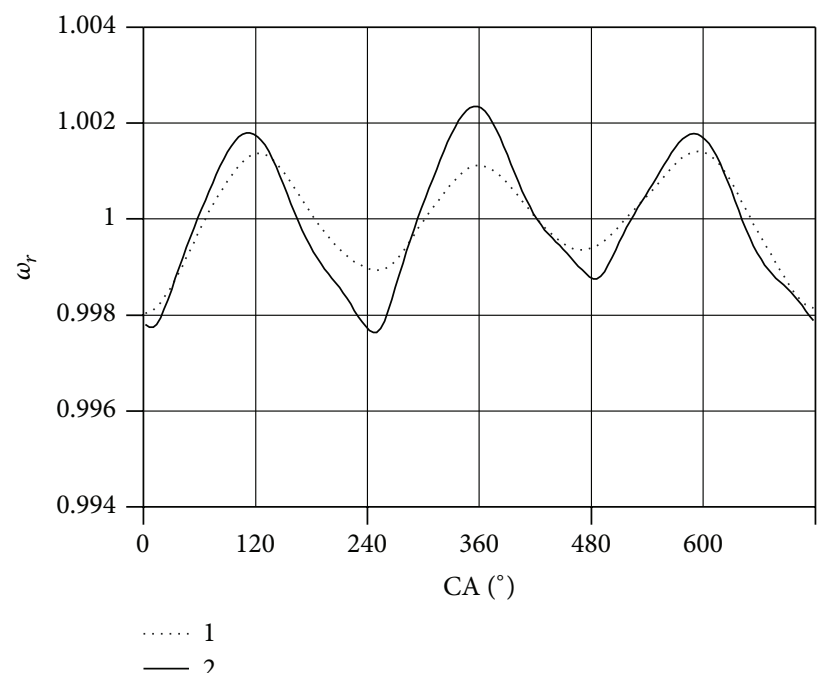

(a)

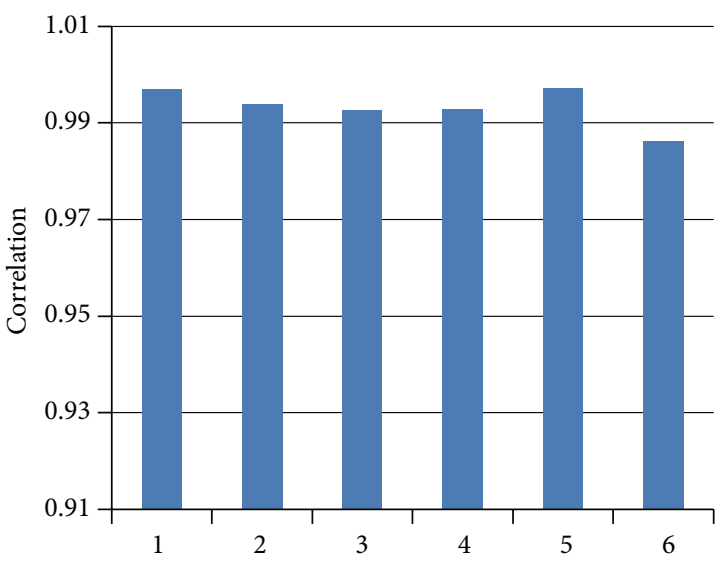

(b)

FIGURE 5: Comparison of smoothed signal (dotted line 1) and model (solid line 2). IAS of healthy engine. Correlation values for $120^{\circ}$ intervals.

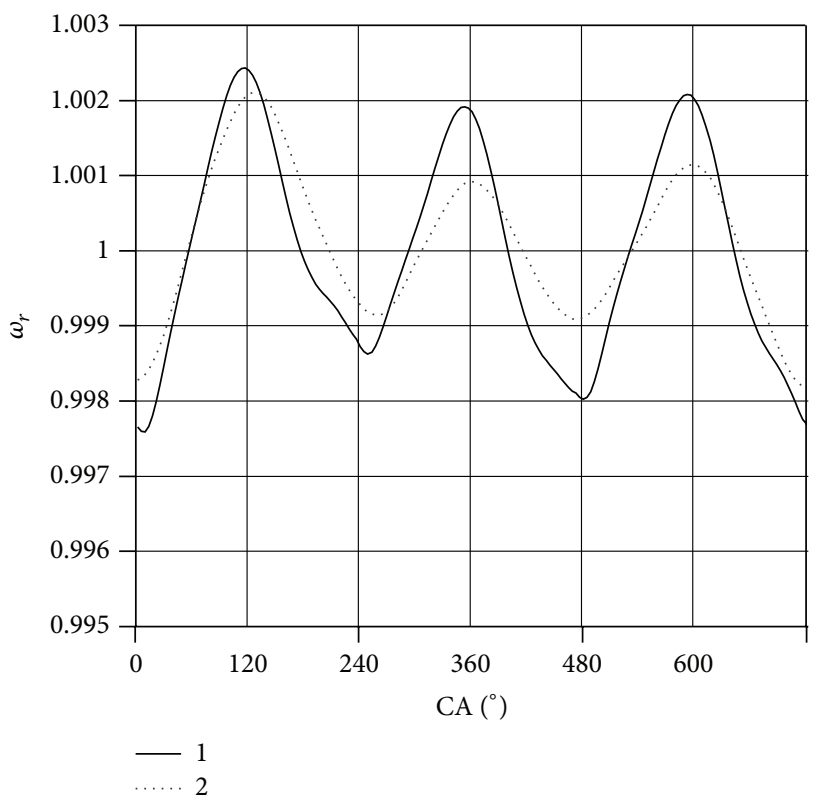

(a)

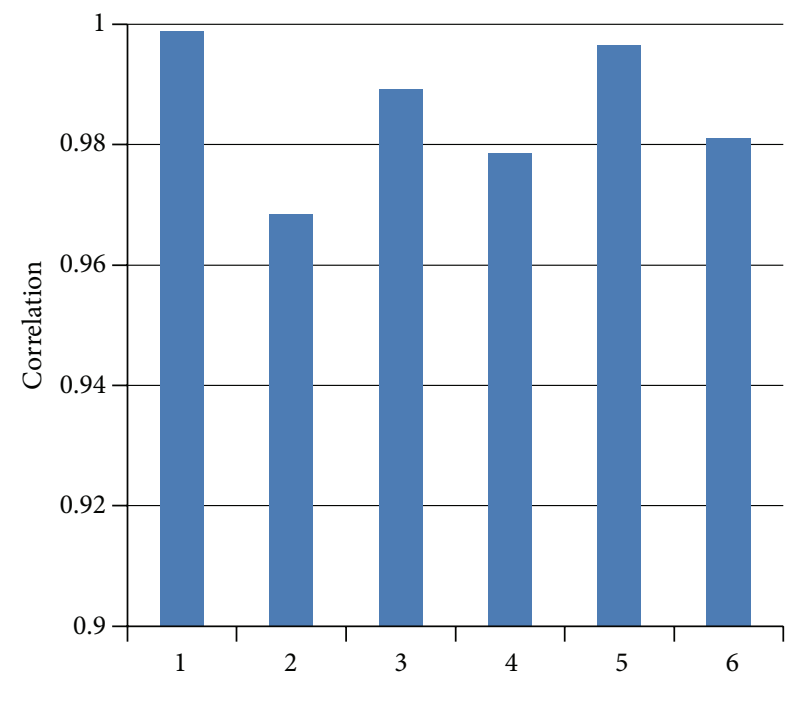

(b)

FIGURE 6: Comparison of smoothed signal (dotted line 2) and model (solid line 1). IAS of engine with failure. Correlation values for intervals' breadth $120^{\circ}$.

Difference between faulty and good conditions was calculated both for measurement and model angular speed. Run of values of $\delta_{\omega}(\theta)$ is presented in Figure 7.

The forms of both functions have positive "+" and negative "-" values in the same intervals. When calculating integral of function $\delta_{\omega}$ in zones of working stroke of every piston, that is, $0-180,240-420$, and 480-660 degrees of crank angle rotation, using

$$
W=\int_{\theta 1}^{\theta 2} \delta_{\omega r}(d \varphi)
$$

next common feature appears, fluctuations of energy of rotation, what is presented in Figure 8.

It shows that in case of fuel leakage, instantaneous angular speed increases in zone previous to affected cylinder and decreases in subsequent zones. The above conclusion is valid 


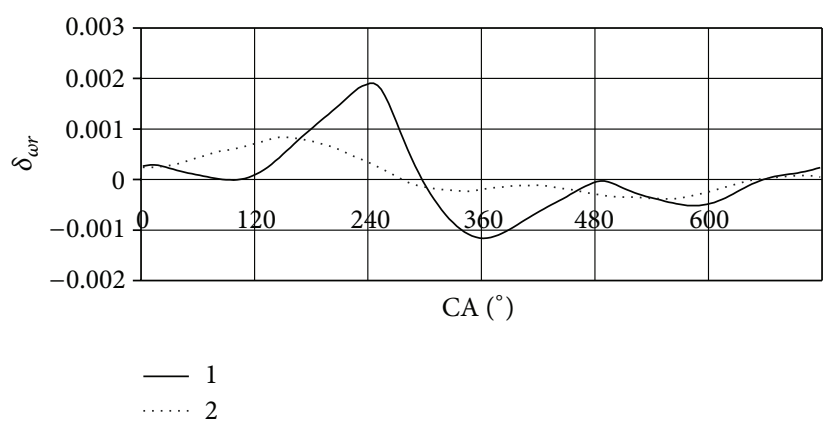

FIGURE 7: Difference of relative angular speed between faulty and good conditions. Model waveform (solid line 1) and measurement waveform (dotted line 2).

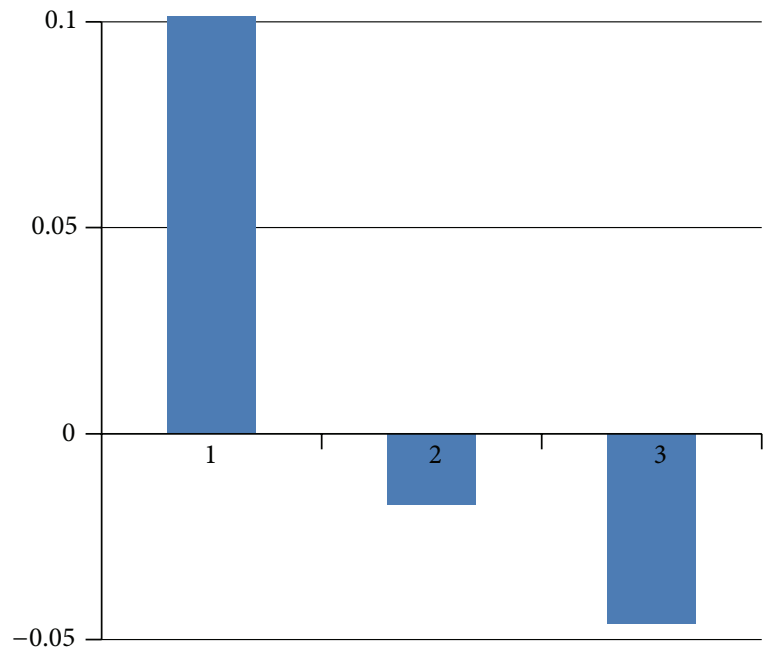

(a)

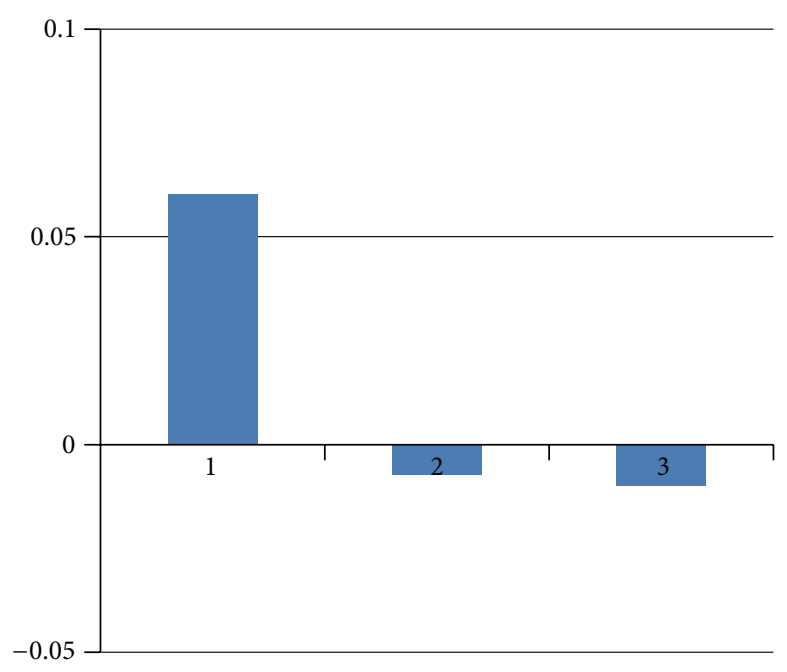

(b)

FIGURE 8: $\Delta$ energy of rotation corresponding to the cylinder number: (a) model, (b) simulation.

for three-cylinder engines and cannot be assumed as general. Important outcome of the above analysis is that model reflects, with acceptable accuracy, the real signal response.

\section{Conclusion}

Analysis presented in this papers lets us assume that SavitzkyGolay filter properties are suitable for instantaneous angular speed signal processing. Achieved results of smoothing gives model and measurement similarity at level sufficient to detect engine fuel system failure. This method can be a base for high pressure fuel system monitoring in practical application. It is clear that implemented malfunction of high pressure fuel pump strongly affects the combustion process and subsequently creates significant changes of angular speed. detection of more "delicate" defects like partly clogged injector sprayer needs to be verified following the methodology presented in this paper.

\section{References}

[1] R. Johnsson, "Cylinder pressure reconstruction based on complex radial basis function networks from vibration and speed signals," Mechanical Systems and Signal Processing, vol. 20, no. 8, pp. 1923-1940, 2006.

[2] J. Franco, M. A. Franchek, and K. Grigoriadis, "Real-time brake torque estimation for internal combustion engines," Mechanical Systems and Signal Processing, vol. 22, no. 2, pp. 338-361, 2008.

[3] M. Geveci, A. W. Osburn, and M. A. Franchek, "An investigation of crankshaft oscillations for cylinder health diagnostics," Mechanical Systems and Signal Processing, vol. 19, no. 5, pp. 11071134, 2005.

[4] K. Cupiał, M. Gruca, and J. Grzelka, "The relationship between the fluctuation of the indicated work, the fluctuation of the crankshaft Speer and the electric current from a generating set," Journal of KONES Powertrain and Transport, vol. 13, no. 2, 2006.

[5] M. Desbazeille, R. B. Randall, F. Guillet, M. El Badaoui, and C. Hoisnard, "Model-based diagnosis of large diesel enginesbased 
on angular speed variations of the crankshaft," Mechanical Systems and Signal Processing, vol. 24, no. 5, pp. 1529-1541, 2010.

[6] P. Charles, J. K. Sinha, F. Gu, L. Lidstone, and A. D. Ball, "Detecting the crankshaft torsional vibration of diesel engines for combustion related diagnosis," Journal of Sound and Vibration, vol. 321, no. 3-5, pp. 1171-1185, 2009.

[7] S. H. Gawande, L. G. Navale, M. R. Nandgaonkar, D. S. Butala, and S. Kunamalla, "Detecting power imbalance in multicylinder inline diesel engine genset," Journal of Electronic Science and Technology, vol. 8, no. 3, 2010.

[8] F. Gu, I. Yesilyurt, Y. Li, G. Harris, and A. Ball, "An investigation of the effects of measurement noise in the use of instantaneous angular speed for machine diagnosis," Mechanical Systems and Signal Processing, vol. 20, no. 6, pp. 1444-1460, 2006.

[9] T. R. Lin, A. C. Tan, L. Ma J, and Mathew, "Estimating the loading condition of a diesel engine using instantaneous angular speed analysis," in Proceedings of the 6th World Congress on Engineering Asset Management, Cincinatti, Ohio, USA, October 2011.

[10] M. Dereszewski, S. Polanowski, and A. Charchalis, "Analysis of diagnostics utility of torque and rotational Speer fluctuation of propulsion shaft of a vessel," Journal of KONES Powertarain and Transport, vol. 18, no. 4, 2011. 


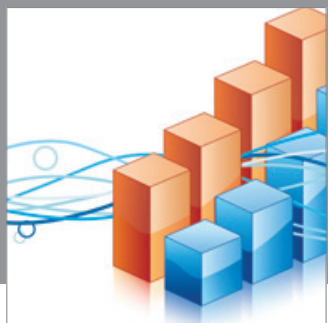

Advances in

Operations Research

mansans

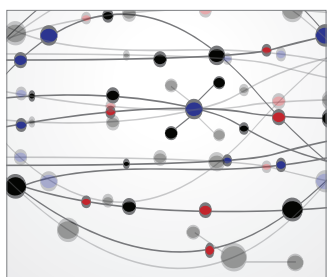

The Scientific World Journal
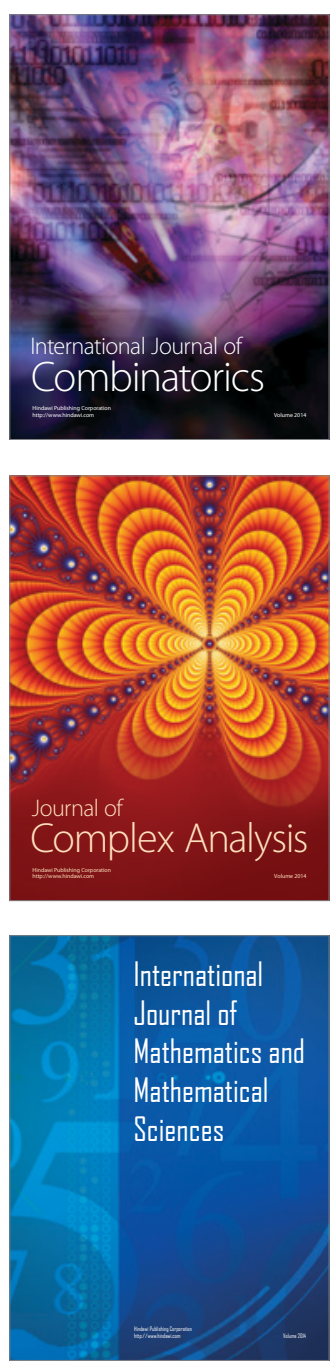
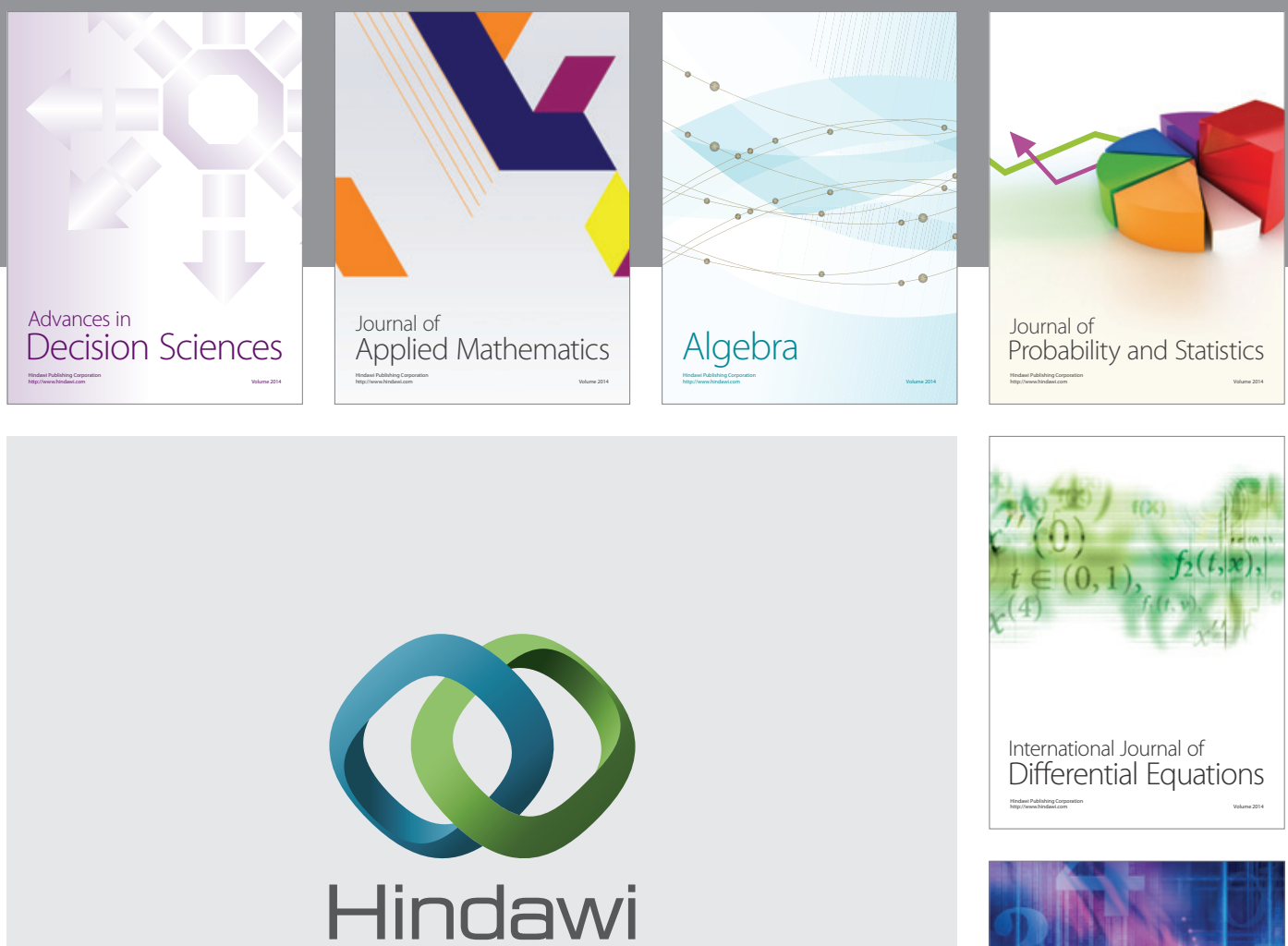

Submit your manuscripts at http://www.hindawi.com
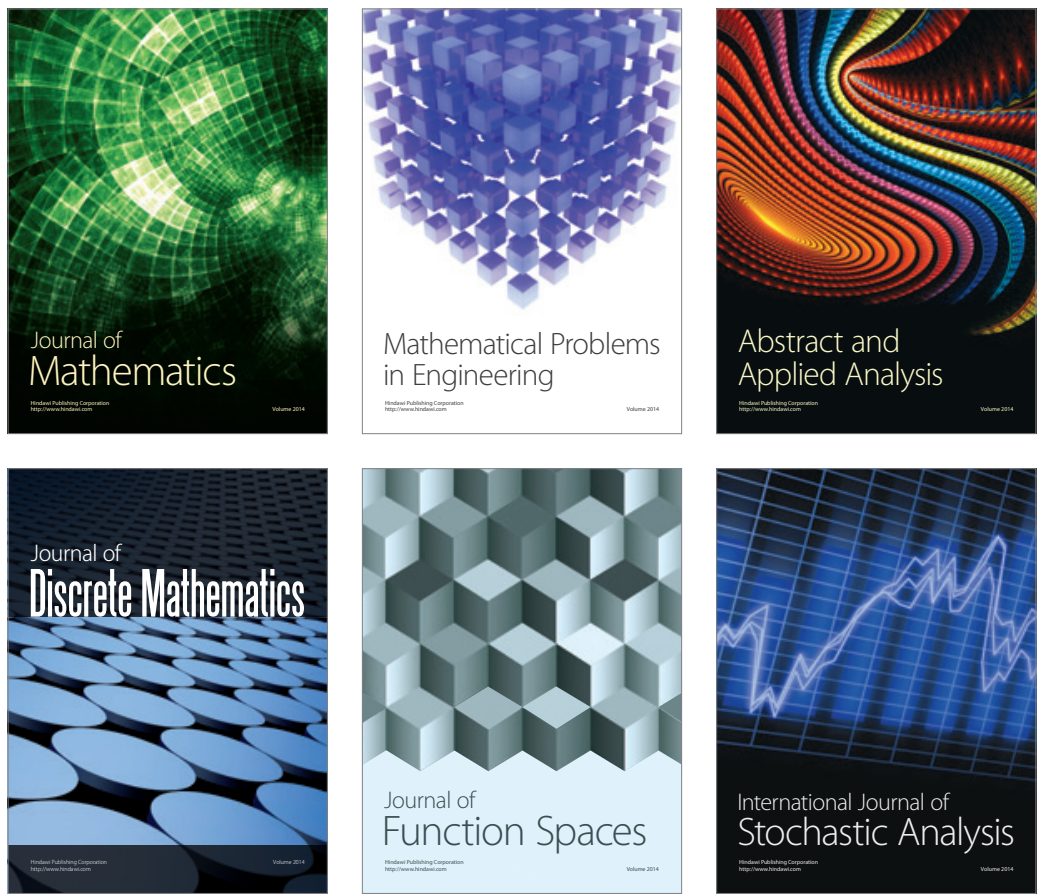

Journal of

Function Spaces

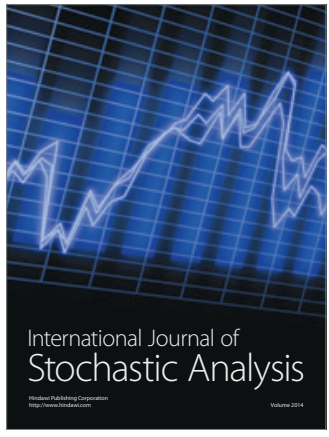

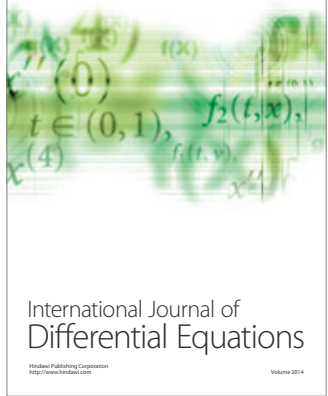
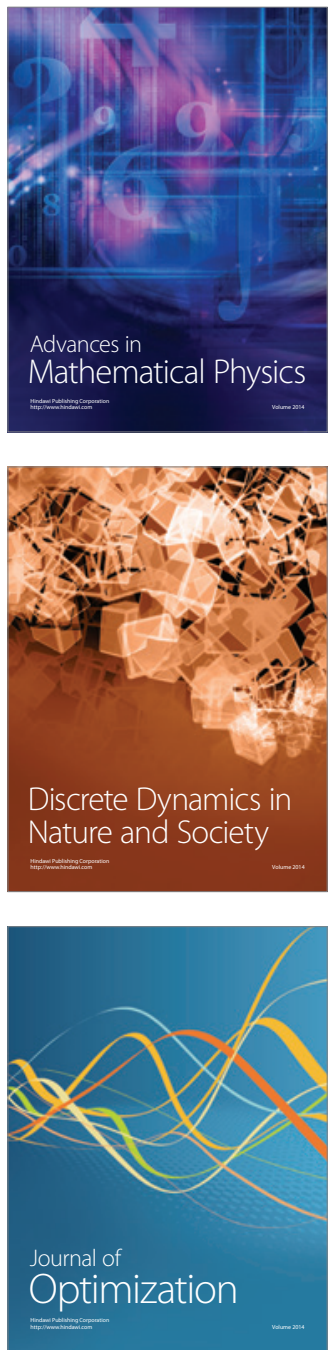